\title{
Ice-Templated Large-Scale Preparation of Two-Dimensional Sheets of Conjugated Polymers: Thickness-Independent Flexible Supercapacitance
}

Jie Zhang ${ }^{\dagger, \ddagger}$, Xueying Fan ${ }^{\dagger, \ddagger}$, Xiaodong Meng ${ }^{\dagger, \ddagger}$, Ji Zhou ${ }^{\dagger, \ddagger}$, Manyun Wang ${ }^{\dagger, \ddagger}$, Shang Chen ${ }^{\dagger, \star,}$ Yawen Cao ${ }^{\dagger,}, Y u$ Chen $^{\nabla}$, Christopher W. Bielawski§,\#, and Jianxin Geng ${ }^{\dagger, \ddagger * *}$

$\dagger$ State Key Laboratory of Organic-Inorganic Composites, Beijing University of Chemical Technology, 15 North Third Ring Road East, Chaoyang District, Beijing 100029, China, E-mail: jianxingeng@mail.buct.edu.cn

$\$$ Beijing Advanced Innovation Center for Soft Matter Science and Technology, Beijing University of Chemical Technology, 15 North Third Ring Road East, Chaoyang District, Beijing 100029, China

${ }^{\nabla}$ Beijing Synchrotron Radiation Facility, Institute of High Energy Physics, Chinese Academy of Sciences, 19B Yuquan Road, Shijingshan District, Beijing 100049, China $\S$ Center for Multidimensional Carbon Materials (CMCM), Institute for Basic Science (IBS), Ulsan, 44919, Republic of Korea

\# Department of Chemistry and Department of Energy Engineering, Ulsan National Institute of Science and Technology (UNIST), Ulsan 44919, Republic of Korea 

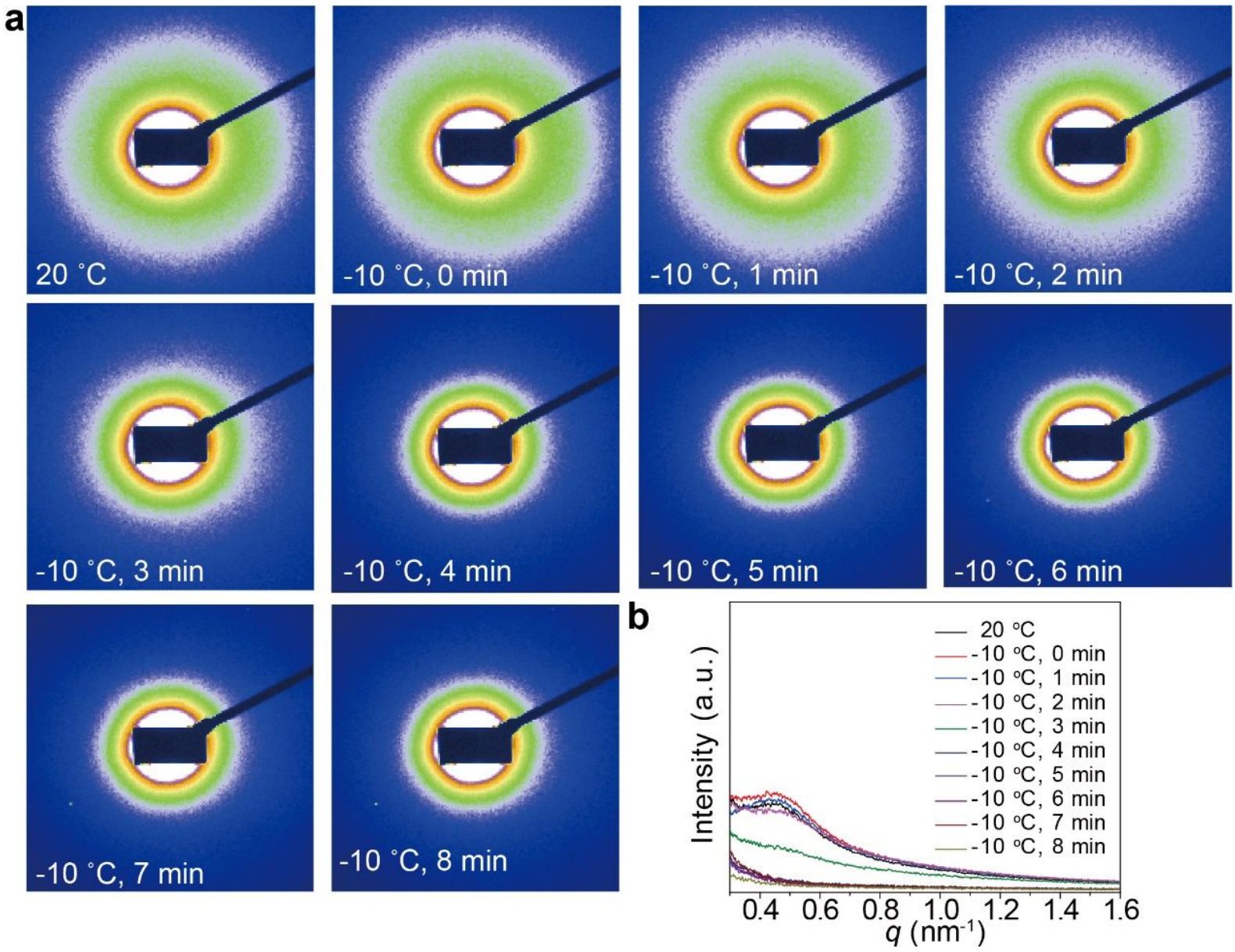

Figure S1. (a) In-situ 2D SAXS patterns recorded at $20{ }^{\circ} \mathrm{C}$ and $-10{ }^{\circ} \mathrm{C}$ from a PEDOT:PSS suspension. The low-temperature patterns were recorded at different times (indicated) after the suspension was rapidly cooled to $-10{ }^{\circ} \mathrm{C}$. (b) Plots of intensity versus $q$ as extracted from the 2D SAXS patterns. 

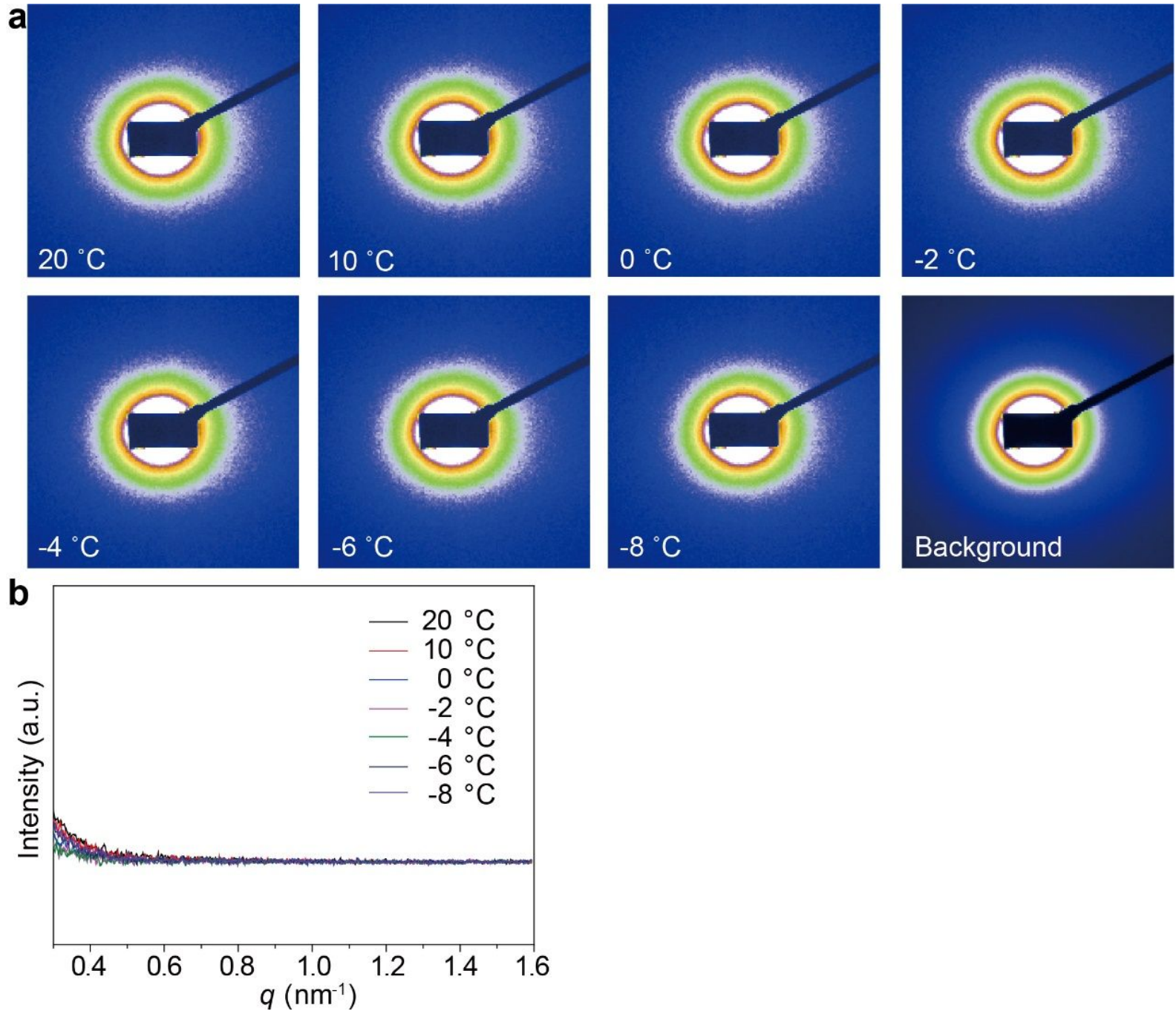

Figure S2. (a) In-situ 2D SAXS patterns recorded from water at different temperatures (indicated) during a freezing process. (b) Plots of intensity versus $q$ as extracted from the 2D SAXS patterns. 


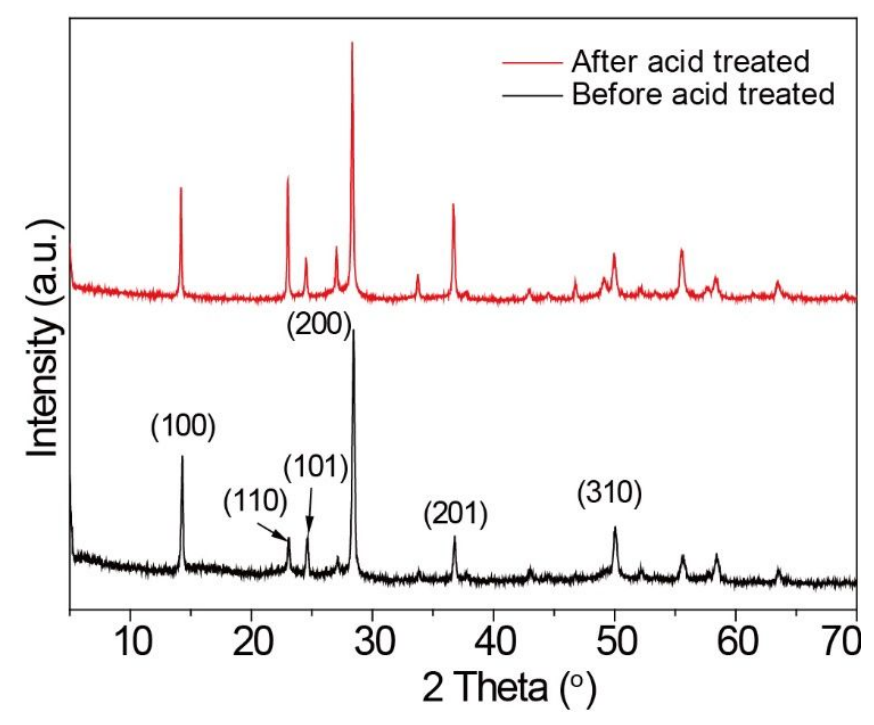

Figure S3. XRD data recorded for $\mathrm{WO}_{3} @$ PEDOT:PSS (bottom) and $\mathrm{WO}_{3} @$ PEDOT (top).
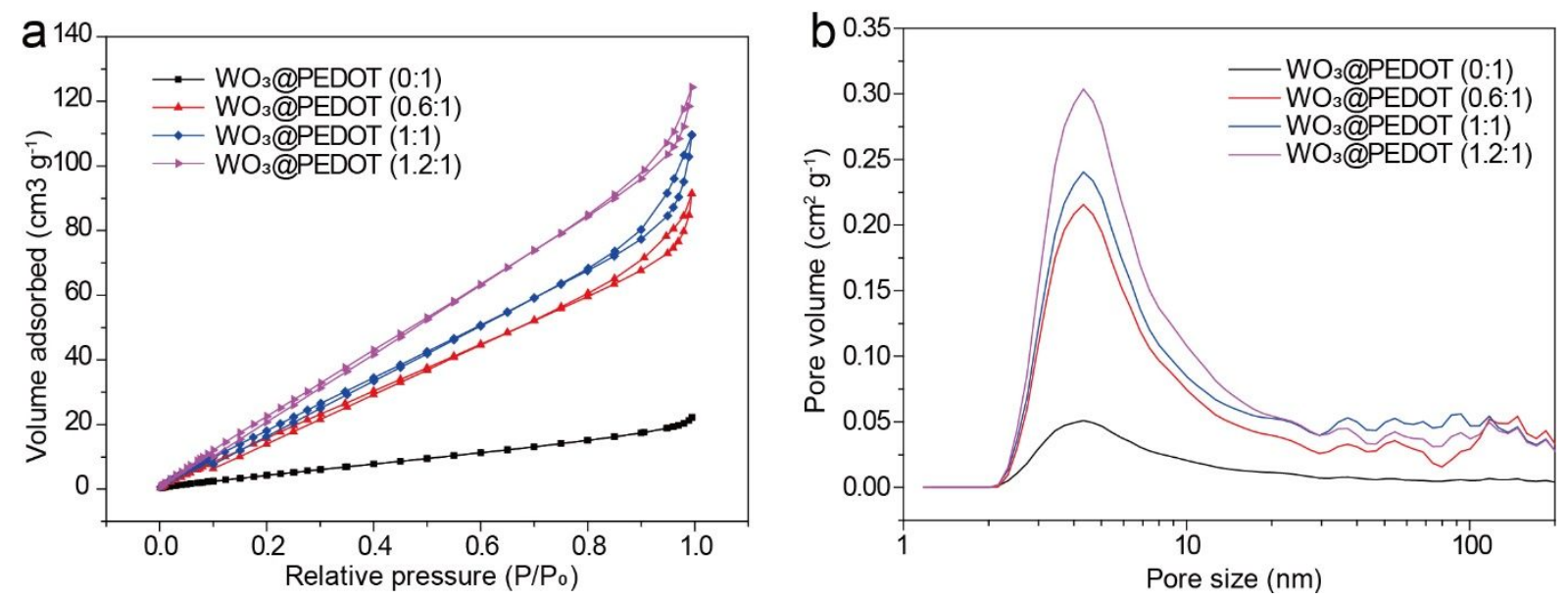

Figure S4. (a) $\mathrm{N}_{2}$ adsorption/desorption isotherms and (b) pore size distributions recorded for PEDOT and the $\mathrm{WO}_{3} @$ PEDOT aerogels obtained at different ratios of $\mathrm{WO}_{3}$ to PEDOT:PSS (indicated). 
Table 1. Specific surface areas of PEDOT and the $\mathrm{WO}_{3} @ \mathrm{PEDOT}$ aerogels obtained at different mass ratios of $\mathrm{WO}_{3}$ to PEDOT:PSS.

\begin{tabular}{cc}
\hline $\begin{array}{c}\text { Mass ratio of } \mathrm{WO}_{3} \text { to } \\
\text { PEDOT:PSS }\end{array}$ & $\begin{array}{c}\text { Specific surface area } \\
\left(\mathrm{cm}^{2} \mathrm{~g}^{-1}\right)\end{array}$ \\
\hline $0: 1$ & 20.9 \\
$0.6: 1$ & 91.4 \\
$1.0: 1$ & 101.9 \\
$1.2: 1$ & 123.1 \\
\hline
\end{tabular}

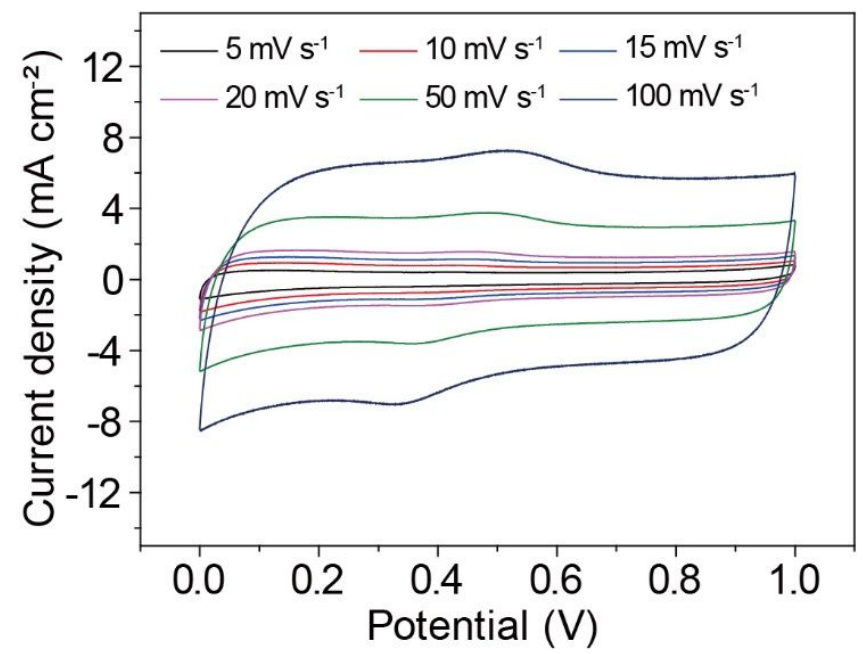

Figure S5. CV data recorded at scan rates that ranged from 5 to $100 \mathrm{mV} \mathrm{s}^{-1}$ (indicated) as obtained from a Swagelok-type cell prepared from a $\mathrm{WO}_{3} @$ PEDOT film with a thickness of $0.25 \mathrm{~mm}$. 

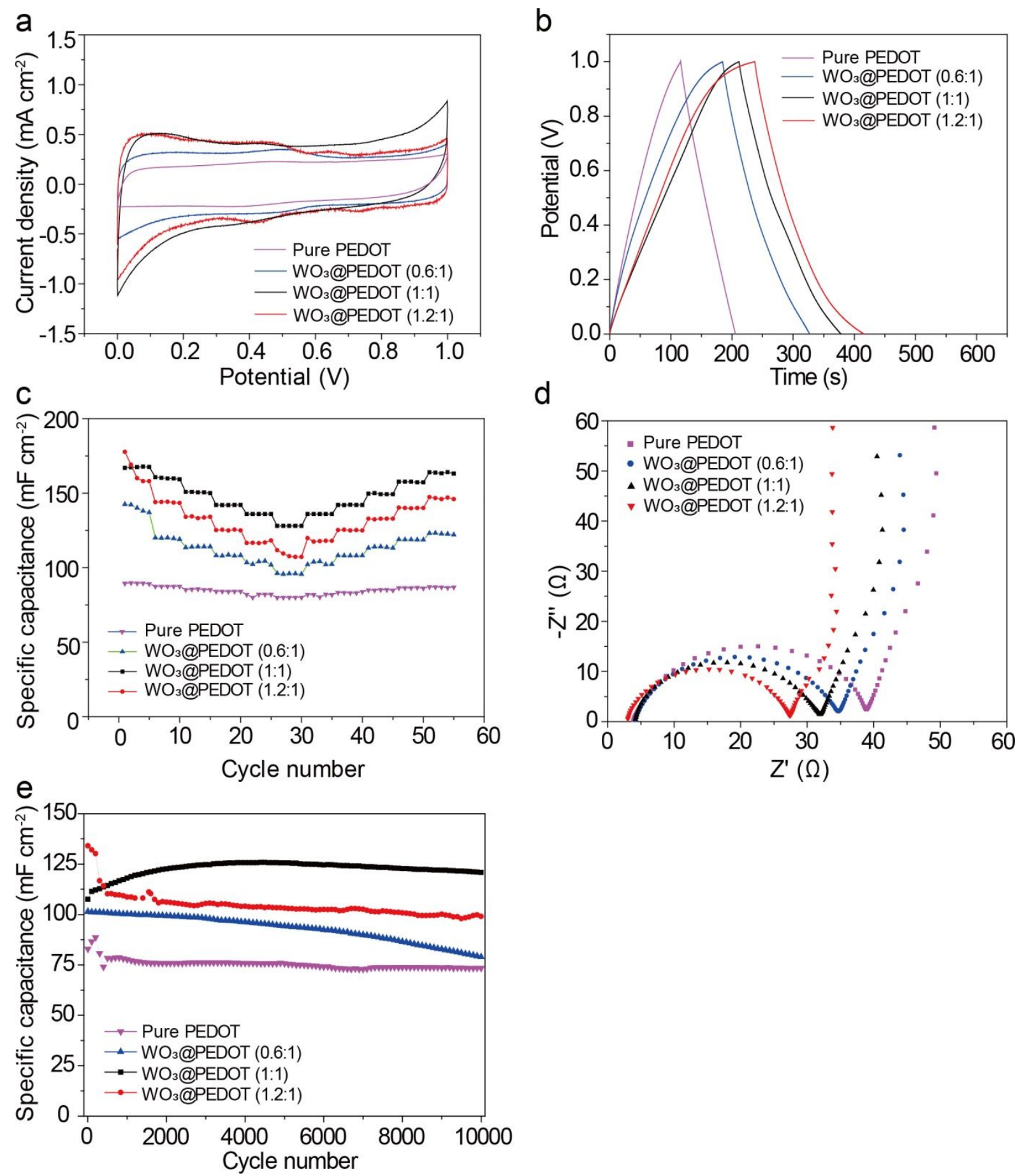

Figure S6. Electrochemical data recorded for supercapacitor cells prepared from different materials (indicated). (a) CV curves. (b) GV charge/discharge profiles. (c) Rate capabilities. (d) EIS curves. (e) Cycling stabilities. All of the supercapacitor cells were prepared using 0.25-mm-thick films. 


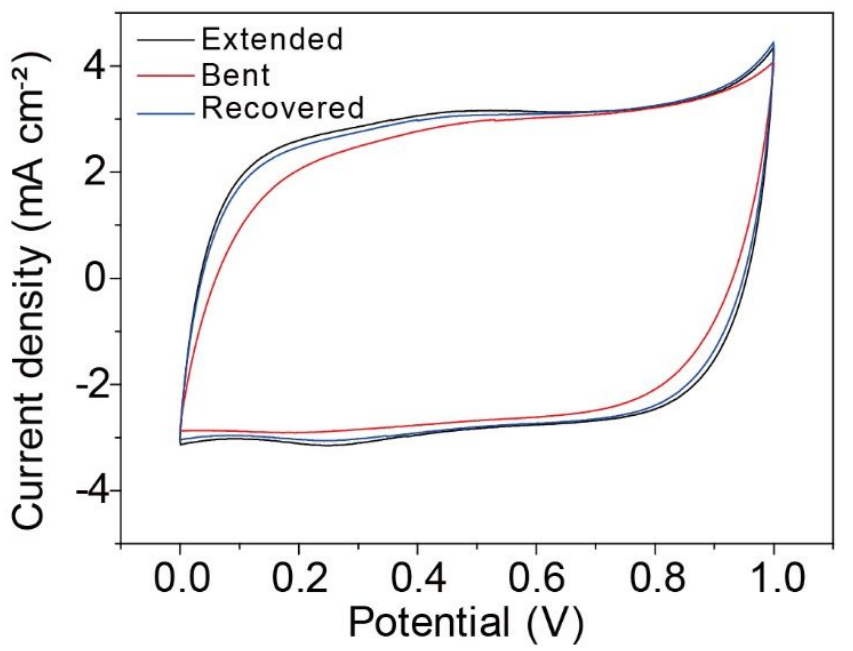

Figure S7. CV data recorded for a pouch-type supercapacitor prepared from a $\mathrm{WO}_{3} @$ PEDOT film with thickness of $0.25 \mathrm{~mm}$ at extended, bent, and recovered states (indicated).

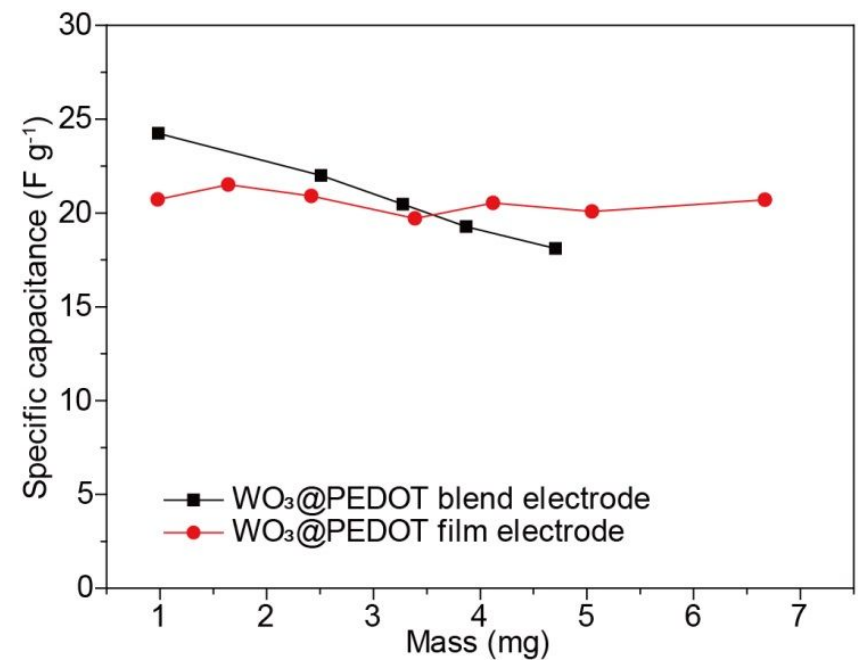

Figure S8. Comparison of the mass specific capacitances recorded for WO3@PEDOT film electrodes and $\mathrm{WO}_{3} @$ PEDOT blend electrodes. 
Table S2. Summary of supercapacitor parameters derived from the Nyquist plots shown in Figure 6k.

\begin{tabular}{ccccc}
\hline Thickness $(\mathrm{mm})$ & $R_{\mathrm{s}}(\Omega)$ & $R_{\mathrm{ct}}(\Omega)$ & $\sigma$ & $D_{\mathrm{H}}^{+}\left(\mathrm{cm}^{2} \mathrm{~s}^{-1}\right)$ \\
\hline 0.125 & 4.0 & 23.8 & 1.5 & $10.4 \times 10^{-6}$ \\
0.250 & 4.2 & 27.5 & 2.1 & $4.8 \times 10^{-6}$ \\
0.375 & 3.8 & 23.6 & 1.8 & $7.2 \times 10^{-6}$ \\
0.500 & 6.5 & 27.1 & 2.2 & $4.6 \times 10^{-6}$ \\
0.615 & 6.5 & 20.0 & 1.9 & $5.9 \times 10^{-6}$ \\
0.750 & 4.7 & 29.6 & 1.7 & $7.4 \times 10^{-6}$ \\
1.000 & 5.1 & 22.9 & 3.0 & $2.4 \times 10^{-6}$ \\
\hline
\end{tabular}

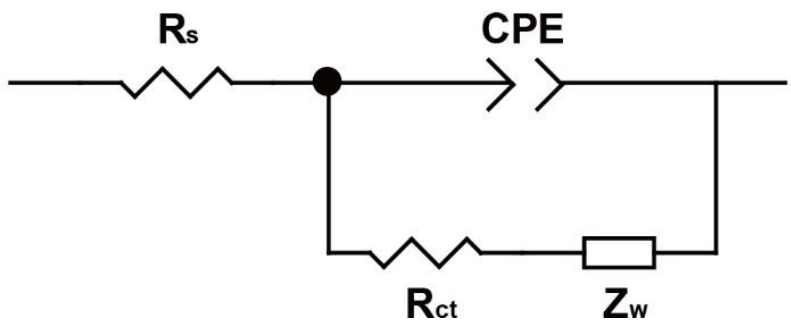

Figure S9. The equivalent circuit used for fitting the Nyquist plots.

Serial resistance $\left(R_{\mathrm{s}}\right)$ and charge-transfer resistance $\left(R_{\mathrm{ct}}\right)$ at the electrode/electrolyte interfaces were obtained by fitting the Nyquist plots in the high-frequency region using the equivalent circuit shown in Figure S9. The diffusion coefficient of $\mathrm{H}^{+}\left(D_{\mathrm{H}}^{+}\right)$was calculated from the Nyquist plots in the low-frequency region using the following formulas: ${ }^{1-3}$

$$
\begin{aligned}
& Z^{\prime}=R_{\mathrm{ct}}+R_{\mathrm{S}}+\sigma \omega^{-1 / 2} \\
& D_{\mathrm{H}^{+}}=\frac{R^{2} T^{2}}{2 A^{2} n^{4} F^{4} C_{\mathrm{H}^{+}}^{2} \sigma^{2}}
\end{aligned}
$$


where $\omega$ is the angular frequency; $Z$ ' is the real component of the impedance and has a linear relationship with $\omega^{-1 / 2} ; \sigma$ is the Warburg factor and can be calculated from the slope of the $Z^{\prime}-\omega^{-1 / 2}$ line; $R$ is the ideal gas constant; $T$ is the absolute temperature; $A$ is the electrode area; $n$ is the number of electrons transferred; $F$ is the Faraday's constant; and $C_{\mathrm{H}}{ }^{+}$is the concentration of $\mathrm{H}^{+}$.

\section{References:}

1. Sun, J.; Xiao, L.; Jiang, S.; Li, G.; Huang, Y.; Geng, J. Fluorine-Doped $\mathrm{SnO}_{2} @$ Graphene Porous Composite for High Capacity Lithium-Ion Batteries. Chem. Mater. 2015, 27, 4594-4603.

2. Aderyani, S.; Shah, S. A.; Masoudi, A.; Green, M. J.; Lutkenhaus, J. L.; Ardebili, H. Comparison of Nanoarchitecture to Porous Media Diffusion Models in Reduced Graphene Oxide/Aramid Nanofiber Electrodes for Supercapacitors. ACS Nano 2020, 14, 5314-5323.

3. Chen, S.; Yang, X. Y.; Zhang, J.; Ma, J. P.; Meng, Y. Q.; Tao, K. J.; Li, F.; Geng, J. X. Aluminum-Lithium Alloy as a Stable and Reversible Anode for Lithium Batteries. Electrochim. Acta 2021, 368, 137626. 